rev.relac.int.estrateg.segur.10(2):21-41,2015

\title{
DISCUSIONES EN TORNO AL ROL ASIGNADO A LA INTELIGENCIA MILITAR EN LA ARGENTINA CONTEMPORÁNEA*
}

\author{
Ángel Pablo Tello** \\ Julio César Spota**
}

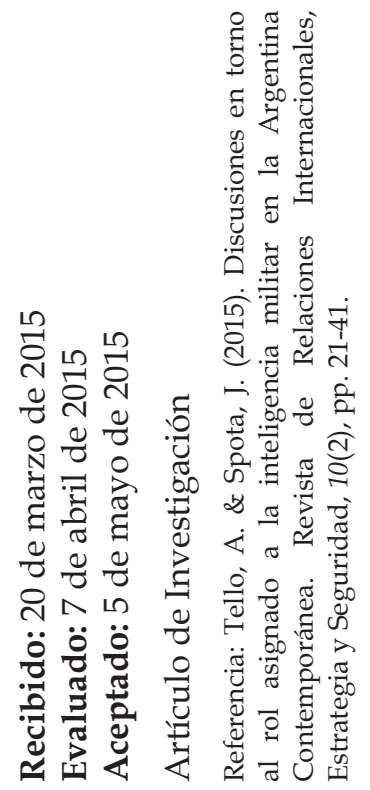

\section{RESUMEN}

En el presente artículo de investigación nos proponemos indagar en la función asignada a la Inteligencia Militar por parte del Estado argentino. El propósito específico del trabajo implicó efectuar un ejercicio de interrogación sobre el papel jugado por la inteligencia militar del país austral dentro del

\footnotetext{
Artículo procedente del Centro de Investigaciones del Instituto de Relaciones Internacionales de la Universidad de La Plata - Argentina y el Instituto de Ciencias Antropológicas de la Facultad de Filosofía y Letras de la Universidad de Buenos Aires - Argentina.

** Profesor Doctor en Relaciones Internacionales, Universidad Nacional de la Plata (UNLP). Docente de Relaciones Internacionales en grado y posgrado. Director de la Maestría en Inteligencia Estratégica Nacional (UNLP). Investigador Universitario en el programa de incentivos y Director de docentes investigadores (UNLP).Correo electrónico: angelpablotello@ hotmail.com

*** Doctor en Antropología, Universidad de Buenos Aires (UBA) y Magíster en Estrategia y Geopolítica, Escuela Superior del Ejército Argentino. Docente de grado (UBA) y posgrado (Escuela Superior del Ejército Argentino y Universidad Nacional de San Martín). Miembro de la carrera de investigador científico de la Comisión Nacional de Investigaciones Científicas y Técnicas (CONICET). Correo electrónico: juliospota@gmail.com
} 
esquema estratégico nacional. A tales efectos encausamos la investigación evaluando el grado de compatibilidad existente entre la apreciación estratégica general manejada por la dirigencia política argentina (caracterizada por una apreciación regional 'pacificada' en términos inter-estatales) y la asignación diferencial de partidas presupuestarias entre los distintos componentes del instrumento militar nacional en beneficio de la Inteligencia Militar. En relación con el plano metodológico articulamos un ejercicio de análisis comparativo de información cuantitativa recabada sobre los presupuestos nacionales -local y regionalesjunto con la realización de una serie de entrevistas efectuadas a personal de inteligencia civil y militar, cuyo eje central fue determinar la interpretación de los acontecimientos en curso desde el enfoque propio de los miembros de los servicios de inteligencia. Los resultados alcanzados nos condujeron a formular una hipótesis: el Estado argentino lleva a cabo actividades ilegales de inteligencia interior utilizando segmentos de la estructura de la Inteligencia del Ejército para lograr su propósito.

Palabras clave: Argentina, Defensa Nacional, Inteligencia Militar, Servicios de Inteligencia, Análisis Comparativo.

\section{DISCUSSIONS ABOUT THE ASSIGNED ROLE TO THE MILITARY INTELLIGENCE IN THE CONTEMPORARY ARGENTINA}

\section{SUMMARY}

The purpose of this research article is to probe the power given by the Argentine State to Military Intelligence. The main goal of the assignment involved inquiring about the role played by the Military Intelligence within the scope of the national strategic management. For purposes thereof, research was indicted assessing the existing degree of compatibility between the general strategic appraisal managed by the Argentinean political management (which can be described as 'pacified' in inter-state terms) and the special budget allocation among the different components of the National Armed Forces to the benefit of Military Intelligence. In the scope of methodology, a comparative analysis study was done, comparing quantitative information taken from national budgets (local and regional ones) and some interviews to intelligence military and civil staff were carried out in order to establish the interpretation of outstanding events by member of Intelligence Services. The final outcome of this research has led to pose a hypothesis for the purpose of historically determining and understanding a set of events which have been taken into account: the Argentine State is carrying out illegal domestic intelligence activities using of the structure of the Military Intelligence in order to fulfill their goals.

Keywords: Argentina, National Defence, Military Intelligence, Intelligence Services, Comparative Analysis. 


\section{DISCUSSÕES SOBRE O ROL ASSINADO À INTELIGÊNCIA MILITAR NA ARGENTINA CONTEMPORÂNEA}

\section{RESUMO}

No presente artigo de investigação propomo-nos indagar na função alocada à Inteligência Militar por parte do Estado argentino. O propósito específico do trabalho implicou efetuar um exercício de questão sobre o papel jogado pela Inteligência Militar dentro do esquema estratégico nacional. A tais efeitos, encausamos a pesquisa avaliando o grau de compatibilidade existente entre a apreciação estratégica geral desenvolvida pela dirigencia política argentina (caracterizada por uma apreciação regional 'pacificada' em termos inter-estatais) e a atribuição diferencial de partidas orçamentarias entre os diferentes componentes do instrumento militar nacional para o benefício da Inteligência Militar. No plano metodológico articulamos um exercício de análise comparativa de informação quantitativa obtida sobre os orçamentos nacionais -locais e regionais- com a realização de uma série de entrevistas efetuadas a pessoal de inteligência civil e militar, cujo eixo central foi determinar a interpretação dos acontecimentos em curso desde o enfoque dos membros dos serviços de inteligência. Os resultados finais atingidos nos conduziram a formular uma hipótese tendente a produzir um entendimento historicamente situado do conjunto de acontecimentos tomados em consideração: o Estado argentino realiza atividades de inteligência doméstica ilegais usando segmentos da estrutura da Inteligência do Exército para alcançar o seu objetivo.

Palavras-chave: Argentina, Defesa Nacional, Inteligência militar, Serviços de Inteligência, Analise Comparativo.

\section{INTRODUCCIÓN}

\section{LA SUPERACIÓN DE OBSTÁCULOS HISTÓRICO-POLÍTICOS COMO PREMISA PARA EL ANÁLISIS DE LA INTELIGENCIA MILITAR ARGENTINA}

En la primera página del prólogo que Zúñiga Berrade le dedicara a la obra Subversión y Seguridad (1970) de Eduardo Crawley, aparece plasmada una impresión personal del autor que, por su naturaleza argumental, asume central relevancia para nuestro estudio. Zúñiga Berrade manifiesta una sentida admiración respecto de la apertura mental característica de los claustros académicos norteamericanos en materia de investigaciones estratégicas. En sus palabras: "descubrí con asombro el gran desarrollo que las entidades estatales y privadas daban a la investigación y difusión de los distintos temas y problemas de estrategia. Es decir, ningún tema estratégico era tabú" (1970, p.7. Negrilla fuera del texto). El talante de la afirmación comprende un principio de libertad de pensamiento y audacia en la investigación como premisas fundantes en el quehacer de la reflexión intelectual. 
Ningún objeto de conocimiento debe escapar a la égida del pensamiento estratégico, a condición de encaminar la pesquisa en cuestión por sendas de rigor metodológico y apego a la evidencia empírica. Acaso el mayor desafío en la República Argentina actual surja en la superación de principios ideológicos operantes en la forma de tamices cognoscitivos. Desde tiempo atrás las lecturas deparadas al estudio de Estrategia Nacional detentan un nivel de involucramiento subjetivista tal que lo tornan incompatible con los requerimientos de objetividad exigidos a un análisis signado por la aspiración de representar la realidad con verosimilitud. Lo dicho impacta con especial énfasis en el campo de la Inteligencia en su función de componente fundamental de la elaboración de una estrategia nacional así como en el proceso de toma de decisión en el estamento político más encumbrado. En repetidas ocasiones, las discusiones suscitadas sobre tópicos inmanentes al terreno de la Inteligencia encuentran mojones de limitación en elementos de contenido ajeno al pertinente a la temática. Nuevamente apropiándonos de las palabras de Zuñiga Berrade, podemos decir que la identidad de las restricciones aparece en la "insistencia y detenimiento en lo ideológico-teórico en perjuicio de lo técnico-práctico” (1970, p.08).

¿A título de qué nos detenemos en exponer la sustancia del perímetro que encajona y desnaturaliza el debate formulado en torno a la Inteligencia a la luz de su relevancia estratégica? El esfuerzo comprensivo orientado a poner de relieve los tan frecuentes como erróneos contornos noestratégicos de la discusión estratégica argentina contemporánea guarda relación con el tema motivador del presente escrito. Habida cuenta del contexto o clima de opinión mayoritario reinante en el campo de la Estrategia y la Inteligencia en la República Argentina, nuestro propósito es analizar sin prejuicio intelectual ni partidario alguno la situación presupuestaria y función práctica de la Inteligencia Militar (IM) en el país. El riesgo cernido en el desarrollo del texto emana de la obvia contemporaneidad del objeto de interés. En la condición 'actual' del tema trasunta el riesgo de anegar el análisis bajo las aguas de la controversia ideológica. La causa remite a la consustanciación existente entre estrategia y política, díada donde la Inteligencia juega una papel preponderante en su calidad de herramienta estatal con magnitud estratégica. Si de acuerdo a un principio de subordinación la estrategia emana y existe por y para el servicio de la política, dada su naturaleza instrumental hablar de estrategia (ámbito intelectual y práctico donde ingresa cualquier cogitación sobre IM) supone, por definición, hablar de política. Y hablar/debatir sobre política conlleva un acto controversial en los tiempos que corren. Máxime cuando la discusión remite a la función de un servicio estatal cuya referencia histórica guarda relación con un tópico tan ineludible como sensible del pasado nacional reciente, a raíz del rol jugado por la IM durante la última dictadura militar (1976-1983) .

1. Sobre la vigencia del particular conviene remitirse a las reflexiones expuestas por Guillermo Holzmann:

La historia del siglo XX en los países de América Latina dejó una imagen perniciosa y perversa del uso de la inteligencia que, se quiera o no, ha terminado cuestionando a los organismos de inteligencia militar en la medida que sus esquemas de organización y doctrina de empleo de la inteligencia constituyeron las bases para la creación de los organismos de Estado y Gobierno que desarrollaren dichas actividades de inteligencia. (Holzmann, 2005, noviembre, p.198. Ver también Ugarte, 1999). 


\section{¿QUÉ ROL LE ASIGNA EL GOBIERNO NACIONAL ARGENTINO A LA INTELIGENCIA MILITAR? LECTURAS ESTRATÉGICAS DE INDICIOS PRESUPUESTARIOS}

Las inquietudes que motivan el presente artículo surgen a la luz del incremento reciente verificado en las partidas presupuestarias asignadas a operativos de radarización desarrollados en vinculación con el área de IM en el noroeste argentino. Las actividades mencionadas representan la prestación de apoyo logístico militar a las tareas de detección y monitoreo de vuelos ilegales en el espacio aéreo argentino, a través de sistemas de radares ubicados en las zonas lindantes con las fronteras internacionales. Los interrogantes nacidos por motivo de los operativos indicados adquieren justificación de cara a la relación establecida entre la visión estratégica nacional, el cuerpo normativo que rige la defensa nacional, el ciclo de planeamiento de la defensa nacional, la función específica del área de IM y el crecimiento presupuestario del área registrado en el Decreto № 1757 (2013) (B.O. 8/11/2013). Esto último, sin el pertinente control de la Comisión Bicameral del Congreso Nacional encargada de monitorear y fiscalizar las actividades de los Servicios de Inteligencia (Tello \& Spota, 2014). En términos generales partimos de los siguientes puntos que hacen la composición del lugar de la estrategia argentina, sin por ello compartirlos en su totalidad:

La perspectiva estratégica nacional se funda en un principio de creciente convivencia pacífica entre los países miembros de la región sudamericana y en una tendencia general en dirección al saneamiento de los diferendos interestatales por vías diplomáticas. El principio democrático cimentado en el imperio del "estado social de derecho" (Vanossi, 1997) a escala regional fundamenta desde lo empírico la evaluación manejada por la dirigencia política. En efecto, de acuerdo a los considerandos de los especialistas en la materia, Sudamérica representa un área con baja la probabilidad de ocurrencia inmediata y mediata de episodios bélicos tradicionales/transestatales. (Bartolomé, 2006; Holzmann, 2010; Vigliero, 2010)

El marco legal que organiza la defensa nacional en la República Argentina instituye una identificación clara respecto de la naturaleza de las amenazas de origen externo, que por motivo de su condición foránea caen en la órbita del potencial empleo del instrumento militar. Según el artículo 10 de la reglamentación de la ley de Defensa Nacional 23.554, se entenderá como 'agresión de origen externo' "el uso de la fuerza armada por un Estado contra la soberanía, la integridad territorial o la independencia política de nuestro país, o en cualquier otra forma que sea incompatible con la Carta de las Naciones Unidas". En otras palabras, el empleo

Cabe destacar que el imaginario social argentino mantiene en plena actualidad la asociación entre IM y represión estatal a pesar de haber transcurrido 31 años desde el retorno de la Democracia. Atentos a que el vínculo resulta injustificado merced del tiempo transcurrido y de la indelegable necesidad estatal de contar con una IM modernizada y eficaz, a lo largo del escrito observaremos que las últimas modificaciones operadas al interior de la IM invitan a meditar sobre los posibles usos ilegales dados al servicio por parte del gobierno nacional desde 2013.

Ángel Pablo Tello - Julio César Spota 
de las Fuerzas Armadas (FFAA) está reservado exclusivamente a repeler y neutralizar acciones armadas propiciadas por unidades estatales. La lógica originaria de la restricción impuesta a la praxis castrense reside en la coyuntura histórico-política atravesada por la República Argentina en la década de 1980. Clausurado el lapso de gobiernos de facto en 1983, el poder político con legitimidad democrática circunscribió la esfera de pertinencia de las FFAA a dimensiones cuya naturaleza impidiese el posible involucramiento del Ejército, la Armada y la Fuerza Aérea en cuestiones políticas. Si bien la Ley de Defensa Nacional data de 1988, fecha que explica su espíritu, la reglamentación fue implementada recién en 2006, momento en que la tesitura nacional en términos de institucionalidad con arreglo a la Constitución cancelaba la posibilidad de asomo alguno de levantamiento armando. Sin embargo la racionalidad rectora de la reglamentación superó las restricciones realizadas en 1988 aunque el clima de época señalase la inviabilidad concreta cobijada en los temores a asonadas militares. En otras palabras, lo que en 1988 resultaba lógico en términos coyunturales fue enfatizado en 2006 en apelación a un análisis anacrónico de la realidad argentina en materia de relaciones cívico-militares (Szeinfeld, 2010; Battaglino, 2013).

El Decreto N 1.728 (2007) donde se estipula el ciclo de planeamiento para la defensa implementó un esquema cuadrienal formulado a los efectos de fijar:

a partir de directivas políticas y criterios técnico-militares, un diseño del Instrumento Militar para el corto, mediano y largo plazo ... cimentado en la metodología de planeamiento por capacidades y, por ende, superador de anacrónicos esquemas basados en hipótesis de conflicto, se erige en torno a los lineamientos fijados en la Directiva de Política de Defensa Nacional. (Decreto Nº 1.714/09)

En sentido contrario a lo dictado por el juicio de especialistas, la República Argentina abandonó el procedimiento de planificación centrado en las hipótesis de conflicto a pesar de que varios de los poderes regionales mantienen dicho principio como eje integrador de su quehacer estratégico ${ }^{2}$. Circunstancia vulneradora de los intereses locales toda vez que la claudicación

2. Por ejemplo Brasil utiliza un eufemismo para enunciar el diseño de sus hipótesis de conflicto: hipótesis de empleo. Las mismas representan "la visión anticipada de posible empleo de las Fuerzas Armadas en determinada situación o área de interés estratégico para la defensa nacional" (Estrategia Nacional de Defensa, 2008. p.46). En la fundamentación del apartado se destaca que Brasil no avizora:

amenazas militares concretas y definidas, representadas por fuerzas antagónicas de países potencialmente enemigos o de otros agentes no estatales [pero debido a la] incertidumbre de las amenazas al Estado, la preparación de las Fuerzas Armadas debe ser orientada para actuar en el cumplimiento de variadas misiones, en diferentes áreas y escenarios, para respaldar la acción política del Estado (Estrategia Nacional de Defensa, 2008, p.47)

En consecuencia, mientras que desde la Casa Rosada se promulga la "planificación por capacidades" indiferente a la lógica estratégica de las formulaciones regionales en materia de Defensa Nacional, y a pesar de las lecturas entusiastas 
en el mantenimiento de la tipología polemológica adoptada en la Argentina restringe sus anticipaciones estratégicas y las fuerza a ingresar en un plano de debilidad programática artificialmente acotado. Si bien la existencia de mecanismos internacionales de transparencia en lo que atañe al gasto militar (GAMIL), como en el caso de la del Centro de Estudios Estratégicos para la Defensa enmarcado en el Consejo de Defensa Suramericano (2014) contribuyen a la estabilización de las relaciones internacionales, es de particular relevancia apreciar la relación comparativa guardada entre el GAMIL Argentino y el resto de los países miembros de la región. Lejos de plantear sugerencias de carreras armamentísticas (Battaglino, 2008) la información de acceso público evidencia una tendencia en los presupuestos militares, traducida en el incremento de los esfuerzos económico-militares entre los diferentes Estados.

En función de la magnitud de su gasto en materia de Defensa, la República Argentina es la tercera unidad estatal regional que menos invierte en GAMIL. Lo anterior se colige al tomar en consideración la representación del GAMIL respecto del PBI de cada estado sudamericano, a condición de incluir en el análisis a Surinam y a Guyana. Excluidos esos países de la medición, la Argentina es el país que dedica la menor cantidad de presupuesto estatal en materia de GAMIL (ver Tabla 1).

Inscrito en el contexto consignado observamos que la IM tiene por fin generar inteligencia vinculada con las posibles amenazas armadas provenientes de otros estados, así como asistir a la dirección militar en la planificación de acciones militares cuando ello fuere requerido. Aceptado lo estipulado ut supra surge un razonamiento de índole paradojal: si la IM cumple la función de producir información vinculada con el Estado, condiciones, posibilidades y probables evoluciones de los instrumentos militares extranjeros, la situación sudamericana indicaría que la teleología justificadora de su propia existencia perdería progresivos grados de justificación, en función de que los países de la región rubricaron acuerdos multilaterales orientados a transparentar el detalle de sus respectivos GAMIL. Entonces, si los poderes participantes de las dinámicas regionales consensuaron la comunicación y fiscalización recíproca en lo tocante al gasto insumido por sus FFAA, ¿Cuál es la razón o justificación para replicar la vigencia del servicio de IM? Dando por sentada la utilidad estratégica de tal servicio y reivindicando su pertinencia como dispositivo estatal indispensable para cualquier formulación estratégiconacional, la pregunta oficia como umbral argumental desde donde plantear la inquietud específica del presente escrito.

generadas al respecto (Eissa, 2013), es un hecho incontestable que el vecino más poderoso en términos militares y económicos de la Argentina (por sólo tomar un ejemplo), esquematiza su diseño de Defensa Nacional con arreglo a previsiones de conflictos donde ingresan de forma indistinta los actores estatales y no estatales como potenciales causales del empleo del instrumento militar. La aclaración adquiere importancia a partir de las limitaciones normativas establecidas para el empleo del instrumento militar argentino. Un eje de discusión que abordaremos en el tercer punto del artículo. 
En aras de completar el cúmulo de información requerido para perfilar el interrogante en toda su complejidad, ocupémonos a continuación de la importancia presupuestaria asumida por la IM en la ley de presupuesto nacional sancionada en 2013. Entre lo presupuestado para 2012 y el 2014 se detecta un incremento del 95,2\% en el área de IM, dato contrastante con la lógica presupuestaria imperante en un GAMIL diseñado por debajo de los estándares internacionales (Spota, 2013). En concreto, los créditos por 1.325 millones de pesos argentinos registrados en las planillas anexas del Decreto № 1757 (2013) prácticamente duplican los 626,9 millones de pesos argentinos que según el Presupuesto Nacional serán destinados a la totalidad del área de Inteligencia Militar en 2012 (ver Tabla 2). En este punto se revela en toda su magnitud la incompatibilidad existente entre la apreciación estratégica general argentina, erigida sobre la 'pacificación' regional sudamericana como suelo axiológico donde se erige la idea de 'planificación por capacidades', la función específica de la IM (ganar conocimiento positivo sobre la situación presente y futuro sobre la situación de los instrumentos militares extranjeros) y el crecimiento presupuestario registrado por la IM en el marco del presupuesto sectorial asignado a la cartera de Defensa. En lo que sigue intentaremos profundizar las implicancias que trasuntadas en la pregunta expuesta en el párrafo anterior y ensayaremos en términos conjeturales algunas interpretaciones que doten de sentido práctico lo que hasta el momento sólo se muestra como una contradicción o contrasentido en los razonamientos.

Tabla 1. Presupuesto Estatal en Materia de Gasto Militar (GAMIL)

\begin{tabular}{|l|r|r|r|r|}
\hline \multirow{2}{*}{ País } & \multicolumn{1}{c|}{$\begin{array}{c}\text { Presupuestos de Defensa (en } \\
\text { millones de dólares) }\end{array}$} & \multicolumn{2}{c|}{ \% del PBI } \\
\cline { 2 - 5 } & Año 2008 & Año 2012 & 2008 & 012 \\
\hline Argentina & $2.830,467$ & $4.003,190$ & 0,87 & 0,84 \\
\hline Bolivia & 258,672 & 335,951 & 1,52 & 1,22 \\
\hline Brasil & $27.540,831$ & $33.665,870$ & 1,67 & 1,41 \\
\hline Chile & $5.019,714$ & $6.095,264$ & 2,80 & 2,27 \\
\hline Colombia & $6.764,721$ & $7.529,000$ & 2,88 & 2,06 \\
\hline Ecuador & $1.914,732$ & $2.662,277$ & 3,15 & 3,29 \\
\hline Guyana & 19,700 & 22,304 & 1,02 & 0,80 \\
\hline Paraguay & 186,854 & 423,040 & 1,00 & 1,63 \\
\hline Perú & $1.622,473$ & $2.047,510$ & 1,28 & 1,03 \\
\hline Surinam & 24,000 & 33,166 & 0,70 & 0,70 \\
\hline Uruguay & 629,429 & 912,551 & 2,07 & 1,84 \\
\hline Venezuela & $3.321,487$ & $4.964,807$ & 1,05 & 1,30 \\
\hline Total & $\mathbf{5 0 . 1 3 3 , 0 8 0}$ & $\mathbf{6 2 . 6 9 4 , 9 3 0}$ & $\mathbf{1 , 7 0}$ & $\mathbf{1 , 4 7}$ \\
\hline
\end{tabular}

Fuente: Centro de Estudios Nueva Mayoría (2013). 
Como fuera estipulado, en atención a la diferenciación programática planteada entre Defensa Nacional (Ley № 23.554) y Seguridad Interior (Ley № 24.059), la Directiva de Organización y Funcionamiento de las Fuerzas Armadas aprobada por Decreto № 1691 (2006) establece que el Instrumento Militar recibe su diseño en el marco de una situación estratégico-regional "de creciente entendimiento y cooperación en materia de defensa y militar". Con lo cual, "el rediseño del Instrumento Militar de la Nación Argentina avanza y consolida el perfil defensivo propio y su disposición cooperativa regional y mundial". A su vez, el punto D del mismo decreto determina el objetivo de controlar con efectividad "los espacios terrestres, marítimos y aeroespaciales de la Nación... en las que se pretende avanzar hacia mayores niveles de cooperación con las naciones vecinas". Ello en dirección a la construcción multilateral de un sistema de defensa subregional de acuerdo a lo estipulado en el punto G del decreto aludido. Más aún, en virtud de lo expuesto en el punto B del Decreto № 1714 (2009), se infiere que el enfoque estratégico organizador de la defensa nacional de la República Argentina abreva en el supuesto que el escenario regional registra una marcada tendencia hacia "El afianzamiento de las alternativas de carácter diplomático y de concertación para la resolución pacífica y negociada de los diferendos y conflictividades interestatales, con explícita renuncia a las alternativas de resolución militar de los mismos".

Tabla 2. Gasto en Inteligencia del Ministerio de Defensa y del Ejército.

Evolución 2007-2013 en millones de pesos

\begin{tabular}{|c|c|c|c|c|c|c|c|c|}
\hline Jurisdicción & 2007 & 2008 & 2009 & 2010 & 2011 & 2012 & $\begin{array}{c}2013(\mathrm{al} \\
26 / 12)\end{array}$ & $\begin{array}{l}\text { Variación } \\
2007- \\
2013\end{array}$ \\
\hline $\begin{array}{l}\text { Ministerio de Defensa } \\
\text { - Presupuesto total }\end{array}$ & 7.421 & 9.098 & 11.391 & 13.792 & 16.849 & 19.127 & 25.487 & $243,4 \%$ \\
\hline $\begin{array}{l}\text { Inteligencia Militar - } \\
\text { Presupuesto }\end{array}$ & 145 & 154 & 220 & 276 & 315 & 384 & 448 & $209,7 \%$ \\
\hline $\begin{array}{l}\text { Estado Mayor General } \\
\text { del Ejército (EMGE) - } \\
\text { Presupuesto total }\end{array}$ & 2.280 & 2.683 & 3.712 & 4.893 & 6.278 & 6.624 & 8.332 & $265,5 \%$ \\
\hline $\begin{array}{l}\text { Jefatura II Inteligencia } \\
\text { Operacional Táctica } \\
\text { del Ejército - } \\
\text { Presupuesto }\end{array}$ & 74 & 88 & 144 & 182 & 211 & 265 & 310 & $317,4 \%$ \\
\hline $\begin{array}{l}\text { Jefatura II - Ministerio } \\
\text { de Defensa }\end{array}$ & $1,0 \%$ & $1,0 \%$ & $1,3 \%$ & $1,3 \%$ & $1,3 \%$ & $1,4 \%$ & $1,2 \%$ & \\
\hline Jefatura II / EMGE & $3,3 \%$ & $3,3 \%$ & $3,9 \%$ & $3,7 \%$ & $3,4 \%$ & $4,0 \%$ & $3,7 \%$ & \\
\hline $\begin{array}{l}\text { Inteligencia Militar / } \\
\text { Ministerio de Defensa }\end{array}$ & $1,9 \%$ & $1,7 \%$ & $1,9 \%$ & $2,0 \%$ & $1,9 \%$ & $2,0 \%$ & $1,8 \%$ & \\
\hline
\end{tabular}

Fuente: ONP (citado por Senado de la Nación, 2013). 
Por otra parte, el artículo 10 de la Ley de Inteligencia Nacional 25.520 establece la creación de la Dirección Nacional de Inteligencia Estratégica Militar (DNIE), con la función primaria de producir inteligencia estratégica operacional y táctica "necesarias para el planeamiento y conducción de operaciones militares y de la inteligencia técnica específica". En resumen, la apreciación estratégico-regional manejada por el Gobierno Nacional señala la existencia de condiciones objetivas y de tendencias políticas intra-regionales orientadas hacia la colaboración inter-estatal entre los poderes sudamericanos ${ }^{3}$. La creciente propensión sudamericana hacia el diálogo constatado en los campos institucional, diplomático y comercial se enlaza con la perspectiva republicano-democrática suscripta por los Estados Nacionales que componen el bloque sub-continental. Así lo estipula el Decreto 727 (2006) que reglamentó la Ley № 23.554 de Defensa Nacional:

la subregión se exhibe ante los ojos del mundo como un ámbito en el cual el equilibrio y el diálogo político democrático aparecen para sus integrantes como una situación consolidada, generando inapreciables ventajas comparativas respecto de otros lugares y/o regiones del mundo.

Siguiendo la línea de razonamiento del Decreto 727 (2006) observamos que las consecuencias en materia de defensa nacional conllevadas por la tesitura internacional, entendidas como escenario estratégico actual y venidero, indicarían una baja probabilidad de ocurrencia en lo concerniente a conflictos armados con los países vecinos, e incluso una menor expectativa en relación a conflagraciones con potencias extra-regionales. Por otra parte lo anterior implica una derivación lógica del abandono de la planificación por hipótesis de conflicto a favor del ciclo de planificación por capacidades, una decisión que acarreó sucesivas distorsiones en el campo de la defensa nacional merced a la hipotética impropiedad estratégica comportada por la postura adoptada. Sostenemos lo anterior no por considerar la inminencia de episodios bélicos interestatales sino porque las secuelas prácticas de la 'planificación por capacidades' fallan en el propósito de lidiar con los desafíos

3. En palabras de Sherman Kent podríamos aludir a la noción de "situación objetiva probable" para definir las lecturas estratégicas pragmáticas, realistas y escindidas -en la medida de lo posible- de voluntarismos. La idea de 'probabilidad', acaso el elemento más problemático en la categoría, remite a una premisa de cuño epistémico: el conocimiento emancipado de cuotas de subjetividad es una aspiración tan deseable como inalcanzable en virtud de que el factor humano ínsito en la operación cognoscitiva representa el involucramiento ineludible de varias instancias de intromisión personalista en la efectuación de un análisis. Como contrapartida la visibilización del componente de parcialización evaluativa restringe el impacto distorsionador producido por la situación particular del analista. En suma Kent reconoce el desvío intrínseco a cualquier apreciación estratégica como resultado ineludible del sesgo innato de las limitaciones y condicionamientos humanos, sin por ello abandonar la apetencia de una descripción basada en fundamentos de aspiración objetivistas (Kent, 1967). La connotación prudencial que trasunta el recaudo conceptual desplegado por Kent nos permite aproximarnos a los escenarios de interés con todo el rigor susceptible de ser instrumentado, a sabiendas de que por definición cualquier evaluación comporta sucesivas instancias de perturbación perceptiva capaz de limitar la validez práctica de las descripciones confeccionadas por los analistas. 
estratégicos presentes y futuros, por introducir a priori un cuota de idealismo wilsoniano incompatible con los análisis pragmáticos en materia estratégica.

La temática gana gravedad frente a la propensión de los poderes regionales a sustentar políticas de estado encaminadas a efectuar gastos sostenidos en el tiempo, o con el fin de modernizar los sistemas de armas y garantizar las capacidades operativas de sus respectivas FFAA. Tendencia en contraste con el desempeño estatal argentino donde sólo el área de IM recibió refuerzos presupuestarios, mientras que el resto de los componentes de las FFAA vieron decrecer en términos reales su importancia presupuestaria. Así, las prospectivas dispuestas por el PEN prefiguran horizontes estratégicos pacificados a causa del diálogo, los acuerdos bilaterales y multilaterales y la inclinación democrática hacia la resolución pacífica de los posibles diferendos inter-estatales suscitados al interior del cono sur. En tanto que el servicio de IM -un elemento estratégico específicamente modelado con la intención de indagar en las intenciones y capacidades militares ajenas- aumenta su predicamento a pesar representar un componente militar cuyo crecimiento contraviene la apreciación estratégica general.

Cabe remarcar que el sentido mismo del servicio de IM y la naturaleza específica de los informes generados en el marco de la DNIE, guardan relación directa con la existencia de amenazas conjeturales o efectivas originadas en el entorno exterior por parte de actores estratégicos estatales. Por ende la integración de los elementos tomados en consideración se traduce en la aparición de una contradicción desplegada entre la mirada estratégica nacional, la presente afectación de partidas presupuestarias incrementadas en el Operativo Fortín II y Escudo Norte en particular y para la Dirección de Inteligencia Militar (DIM) en general. Pues el enfoque estratégico nacional, un esquema analítico de alcance local, regional y global donde se inserta la lógica de la Defensa Nacional in toto, anticipa la configuración de contextos internacionales inmediatos y mediatos carentes de conflagraciones. Nos encontramos entonces ante una paradoja puesto que al ser articulados los elementos presentados se impone un cuestionamiento de base: ¿Cuál es el sentido último del crecimiento presupuestario verificado en el área de la IM si en términos estratégiconacionales, la República Argentina avizora un futuro cercano sin amenazas armadas por parte de potencias extranjeras y el gasto militar de los países sudamericanos está transparentado a partir de los acuerdos que dieron forma a la UNASUR?

Las respuestas factibles de ser prefiguradas se inclinan por: a) la existencia de un principio de incoherencia en lo concerniente a la relación entre la evaluación estratégica general de índole 'pacificada', y la acción de la IM en calidad de elemento sectorial cuyo propósito es anticipar amenazas externas; con la concomitante utilización ineficaz de recursos estatales en un área cuya relevancia iría disminuyendo de la mano del aparente decrecimiento de las probabilidades de amenazas externas; b) Una moción de re-funcionalización en el área de Inteligencia Militar con objeto de asignarle un papel diferente al fijado en el cuerpo de normas que dictamina su área de competencia y acción y c) una causa no anticipable. Cualquiera sea el motivo subyacente a las medidas presupuestarias efectuadas, hubiera sido necesario abrir el debate sobre los fundamentos últimos que apuntalan la decisión a fin 
de garantizar una utilización racional de los recursos estatales dispuestos a tales efectos, fiscalizar el apego del área de IM a lo fijado en el marco legal que establece la naturaleza de su desempeño y, en suma, tornar inteligible una decisión presupuestaria que -en su aspectocontraría la lógica del enfoque estratégico nacional en materia de defensa. Pongamos en suspenso la profundización de la temática para ocuparnos a continuación, y con la brevedad del caso, de una discusión vinculada con las derivaciones prácticas de un elemento clave del ordenamiento jurídico de la defensa nacional: el Decreto de Reglamentación de la Ley de Defensa Nacional como marco de discusión sobre el potencial involucramiento de las FFAA en la lucha contra el narcotráfico.

\section{FALLAS ESTRUCTURALES COMO ORIGEN DE PROBLEMAS COYUNTURALES}

El desarrollo argumental previo apuntó a poner de relieve la incompatibilidad existente entre los esquemas estratégicos generales diseñados por la dirigencia política contemporánea y su instrumentación empírica en lo tocante a una de sus reparticiones clave: la IM. Los ejemplos abundan. Y el discutido aquí constituye tan sólo una muestra representativa dentro de una tendencia general hacia los desaciertos en materia de defensa nacional sucedidos en el último decenio. Para apreciar una discontinuidad incluso más extensiva basta volverse hacia otro aspecto de la reglamentación de la ley 23554 de Defensa Nacional elaborada en 2006 mientras la Dra. Nilda Garré ocupaba la cartera, para demostrar la existencia de una contradicción operativa nacida en los desfasajes plasmados al interior del plano normativo. La inconsistencia aludida media entre un instrumento legal otrora promocionado por este gobierno y lo que hoy (con aparente ánimo de urgencia) se pretende impulsar en la forma de medidas descoordinadas. En particular nos referimos al contraste que se advierte entre la mencionada reglamentación y una medida estratégico-operativa actual según la cual el Gobierno Nacional involucra a las FFAA con un grado de creciente protagonismo en la lucha contra el narcotráfico. Este último se trata de un fenómeno que en seguridad internacional es conceptualizado como una "nueva amenaza" (Bartolomé, 2006; 2010; Sepúlveda, 2007). Empero, entre los considerandos de la reglamentación traída a colación aparecen impedimentos explícitos contra la realización de un emprendimiento de la índole relatada.

Deben rechazarse enfáticamente todas aquellas concepciones que procuran extender o ampliar la utilización del instrumento militar hacia funciones totalmente ajenas a la defensa, usualmente conocidas bajo la denominación 'nuevas amenazas', responsabilidad de otras agencias del Estado organizadas y preparadas a tal efecto; toda vez que la intervención regular sobre tales actividades supondría poner en severa e inexorable crisis, la doctrina, la organización y el funcionamiento de una herramienta funcionalmente preparada para asumir otras responsabilidades distintas de las típicamente policiales.

El dilema de fondo de la propia noción de 'planificación por capacidades' radica en la profundización conceptual de la diferencia legal vigente entre las esferas de la seguridad interior y la defensa nacional. Esta distinción constituye un principio de categorización 
mutuamente excluyente cargado de sentido histórico y político cuando fuera sancionada en 1988 pero requerido de actualización en los tiempos que corren y de la superación de los inconvenientes acarreados por su reglamentación. La limitada viabilidad práctica derivada de las restricciones operativas impuestas por la reglamentación en vigencia, instituyen un rango de aplicación artificialmente limitado de cara al formato de los retos contemporáneos. Frente a la multidimensionalidad e imbricación de las amenazas cernidas en la actualidad y el porvenir, continuar manteniendo el esquema de seguridad interior y defensa nacional como esferas radicalmente diferenciadas entre sí supone un intento ímprobo de forzar la realidad dentro de la teoría. Vale recordar que en su momento de sanción la ley respondió con solvencia a la coyuntura histórica imperante en el país. Pero casi un cuarto de siglo después lo que otrora significó un acierto legislativo en relación al momento vivido en 1988, en la actualidad supone una moción desacertada por mor de la evaluación estratégica imprecisa que sustentó su reglamentación del 2006. Tal situación conlleva subordinar lo empírico a las abstracciones ideologizadas, un rasgo político que parecería característico de la dirigencia actual.

En su conjunto la actual percepción estratégica nacional junto a su correlato en materia de Defensa Nacional produce el germen de inadecuación del que nacen las inconsistencias como la discutida a lo largo de este artículo. Más todavía, la misma tónica de 'planificación por capacidades' refiere a una postura estratégica anacrónica, ya criticada en el Informe Rattenbach redactado a propósito de la derrota ocurrida como resultado de la Guerra de Malvinas. Por otra parte, al combinar el imperativo de exclusión entre seguridad interior y defensa nacional con las implicaciones inherentes a la definición de la 'planificación por capacidades' tienen lugar eventos de invisibilización de aspectos que competen al bienestar de la población. En concreto, la sintonía de la 'planificación por capacidades' dictamina que el terrorismo internacional, una amenaza que en Argentina -por ejemplo- se tradujo en el atentado contra la AMIA perpetrado en 1994, no sería objeto de interés para la defensa nacional por tratarse de una agresión cometida por actores no estatales. A su vez el contenido conceptual de la 'planificación por capacidades' cobijado en la reglamentación prescribe la acción de las FFAA en el combate contra las 'nuevas amenazas', siendo el narcotráfico una de ellas y quizás la más relevante para la agenda contemporánea de seguridad argentina.

Sin embargo, el creciente rol jugado por las FFAA que en apariencia podría llegar a superar el cumplimiento de la función de apoyo logístico asignada en los operativos como Escudo Norte y Fortín II, objeta la aceptación gubernamental de sus propios considerandos. Sucede que la naturaleza híbrida de las nuevas amenazas demanda soluciones creativas y actualizadas al formato específico presentado por el desafío estratégico que representan. Empeorando el panorama, todo indica que la conducción política nacional no atina a dar cuenta de la complejidad del problema por atarse a una visión estratégica nacional estrecha de miras, a la impropiedad radicada en las limitaciones legislativas autoimpuestas en la reglamentación de la ley de Defensa Nacional y a la falta de coordinación orgánica entre las herramientas del Estado. La vastedad del problema erigido por las 'nuevas amenazas' exige la construcción de 
una solución integral donde ingresen en igual medida la corrección de los errores actuales y una revisión general de reglamentación de la Ley de Defensa Nacional, junto a la formulación de esquemas estratégicos nacionales capaces de lidiar con las amenazas reales cifradas en el horizonte de posibilidades abierto para la República Argentina en el siglo XXI.

\section{DISCUSIÓN. APRECIACIONES CONJETURALES SOBRE LA FUNCIÓN ASIGNADA A LA INTELIGENCIA MILITAR EN LA ARGENTINA CONTEMPORÁNEA}

Retomando el hilo de la reflexión nodal del trabajo surge la necesidad de interrogar la situación con la esperanza de otorgarle racionalidad al escenario bajo análisis. En concreto, ¿qué explicación podemos otorgarle a una situación como la descrita? ¿Cómo tornar inteligible la contradicción expuesta entre la concepción estratégica general argentina cimentada en el entendimiento de la región sudamericana como área pacificada en términos interestatales, en simultáneo con la verificación del incremento presupuestario ocurrido en el servicio de IM, cuando esta última funciona como herramienta estatal específicamente diseñada para conocer las capacidades militares extranjeras? Intentar una conciliación entre los elementos antagónicos supone un esfuerzo conjetural ímprobo puesto que caemos en la cuenta de la aparente inviabilidad lógica de la tarea. En otras palabras ¿Para qué potenciar la capacidad de indagar en un aspecto como el poder de las FFAA de los países vecinos si el Estado argentino afirma no tener hipótesis de conflicto? El interrogante aumenta en extrañeza al tomar en cuenta la existencia mecanismos de transparencia en lo concerniente al GAMIL encaminados por vía de organismos multilaterales. Entonces ¿cuál es la intención real detrás de las decisiones tomadas por el poder político? En la forma de hipótesis de trabajo nos manejamos con dos afirmaciones susceptibles de corroboración: A: el Estado Nacional malgasta recursos al sancionar incrementos presupuestarios para áreas de importancia secundaria en su esquema estratégico general 'pacificado'; y B: la asignación de partidas presupuestarias incrementadas en IM adquiere sentido en la intención del Estado Nacional de perseguir fines no previstos en la leyes ordenadoras del área de la inteligencia. Con objeto de ampliar las miras del análisis expusimos las mencionadas hipótesis a oficiales de IM y agentes del resto de los servicios de inteligencia durante sucesivas entrevistas en búsqueda de información capaz de esclarecer las inquietudes que motivaron el presente escrito.

La pesquisa, realizada a condición del rutinario anonimato de las fuentes, arrojó como resultado una apreciación coincidente entre la mayoría de los informantes consultados. Según las versiones recabadas el Poder Ejecutivo Nacional (PEN) habría perdido confianza en la Secretaría de Inteligencia (SI), el organismo centralizador y articulador de las actividades de inteligencia en la Argentina, a causa de la provisión de información política distorsionada adrede. Ocurre que en 2013 fueron celebradas las elecciones de medio término donde tiene lugar un recambio parcial en la conformación del parlamento, precedido por las elecciones Primarias Abiertas Simultáneas Obligatorias (PASO) donde los partidos políticos dirimieron sus respectivas candidaturas. De acuerdo a las fuentes inquiridas el PEN generó un requerimiento 
a la SI para averiguar si Sergio $\mathrm{Massa}^{4}$, un popular candidato -ahora opositor pero otrora oficialista- que barajaba la posibilidad de presentarse en la compulsa como opción electoral opositora, efectivamente se presentaría en los comicios.

El requerimiento de marras habría sido efectuado a sabiendas de que su formulación implicaba una violación contra la Ley Nacional de Inteligencia (25.520) que en su artículo 4, incisos 2 y 3 prohíbe la obtención, producción y almacenamiento de inteligencia sobre individuos por su identidad religiosa, étnica o política. Asimismo está vedada la gestación de influencias institucionales y el involucramiento en la vida de los partidos políticos. Siempre en atención a lo relatado por las fuentes, el producto de inteligencia ilícito entregado al PEN sostenía que Massa no poseía intención alguna de involucrarse en el proceso electoral. La posterior presentación de Massa, su consiguiente victoria en las PASO y las concomitantes elecciones parlamentarias provocaron la ruptura del nexo de confianza tendido hasta entonces entre el PEN y la SI, dado que el primero evaluó intencional la provisión de inteligencia errada por parte del segundo ${ }^{5}$. El desmoronamiento del lazo de confianza habría conducido al PEN a valerse de elementos de Dirección de Inteligencia Estratégica Militar para ejercer funciones al interior del país (algo también prohibido por la ley rectora del área). En una reciente intervención periodística aparecida en el matutino La Nación, Joaquín Morales Solá coincidió con el razonamiento expuesto hasta el momento:

la Presidenta se enojó con los espías de la ex $\mathrm{SIDE}^{6}$. No le daban buena información política (lo que es cierto) y, encima, dejaron de influir en importantes despachos judiciales. El rumor señala que los espías civiles se molestaron con Cristina Kirchner cuando ésta firmó el acuerdo con Irán por el que se comprometió a intercambiar información sobre la masacre en la AMIA. Ese intercambio podía exponerlos, dedujeron, o exponer a sus fuentes en el exterior. (Morales, 2013, 23 de diciembre)

La hipótesis manejada aquí -cónsona con el desarrollo aportado por Morales Solá- recibe contextualización en un momento en que el PEN designó como jefe del Ejército argentino a César Milani, un oficial perteneciente a la IM con cuestionamientos por parte de las

4. Sergio Massa se desempeñó como Director General de la Administración General de Seguridad Social (2002-2007), Intendente de Tigre (2007-2008, 2009-2013), Jefe de Gabinete de la Presidencia de la Nación (2008) y diputado Nacional por la Provincia de Buenos Aires (2013 en adelante). En la actualidad dirige el partido Frente Renovador desde donde se postula como candidato a Presidente de la República de cara a las elecciones a ser celebradas en octubre de 2015.

5. En el léxico propio del sistema de inteligencia argentino la acción de proporcionar información viciada con objeto de confundir al consumidor se denomina "vender pescado podrido".

6. La actual SI otrora poseía la denominación de Secretaría de Inteligencia del Estado (SIDE), nombre que aún continúa en uso coloquial. De allí que el Morales Solá haga mención de la "ex SIDE". 
agrupaciones defensoras de los Derechos Humanos (DDHH). La impugnación, compartida aún por los organismos de DDHH afines al gobierno nacional ${ }^{7}$, nace ante la existencia de evidencia probatoria que vincula a Milani con violaciones a los DDHH durante la última dictadura militar argentina. El papel jugado por el actual jefe del Ejército argentino implicaría el manejo de un aparato de inteligencia paralelo al oficial montado sobre la una parte de la estructura de la IM. El organismo fuera de la ley habría recibido la tarea de efectuar inteligencia dentro del territorio nacional y centrado en el seguimiento de líderes políticos, sociales, empresariales y sindicales enfrentados con el gobierno nacional. El parecer de Rosendo Fraga refuerza la verosimilitud de los informes recabados durante las sucesivas entrevistas mantenidas con las fuentes antes mencionadas y con la tónica de los datos proporcionados por Morales Solá:

\begin{abstract}
Milani viene cumpliendo también un rol extraoficial en materia de inteligencia interior a pedido del gobierno, como lo han denunciado legisladores opositores. Como Jefe de Inteligencia vio incrementar fuertemente su partida secreta para estas actividades. Ya al frente del Ejército, en noviembre de 2013, el Ejecutivo dio 1300 millones de pesos adicionales para realizar "tareas de apoyo contra el narcotráfico". Es una asignación extrapresupuestaria cuyos fondos se gastan fuera del presupuesto y en forma discrecional. Esta cooperación se viene dando desde hace más de dos años en el marco del Operativo Fortín que se realiza en el norte del país, con apoyo de efectivos del Ejército y medios de Fuerza Aérea. (Morales, 2013, 23 de diciembre)
\end{abstract}

Son numerosas las acusaciones contra Milani por actividades de Inteligencia ilegal y provienen de actores políticos y sociales con diferentes orígenes, funciones y trayectorias. Sólo a título representativo y con objeto de no abundar en el particular podemos hacer mención de las palabras del Senador Nacional por la Provincia de Jujuy Gerardo Morales, quien afirmó que "Milani viene haciendo tareas de inteligencia interior sobre el periodista Daniel Santoro" (23 de diciembre, 2013). De forma supletoria a Morales el Diputado Nacional por La Rioja Jorge Martínez (23 de diciembre, 2013) sostuvo no existen duda que la 'refuncionalización' del Ejército al servicio del "proyecto nacional y popular"8 tiene como principal objetivo su participación en actividades de inteligencia y seguridad interior, ambas vedadas por la ley de defensa, la ley de seguridad interior y la propia ley de inteligencia. Y profundizó su crítica al evocar la inhibición legal manifiesta que pesa sobre dichas actividades: "la participación de las FFAA en tareas de seguridad interior está explícitamente vedada por la legislación vigente, excepto en casos muy puntuales y dentro de los precisos límites y criterios establecidos por la Ley de Seguridad Interior No. 24.059" (Martínez, 2013, 23 de diciembre).

7. Tal es el caso del Centro de Estudios Legales y Sociales (CELS).

8. Dicha denominación es uno de los lemas esgrimidos por el partido político encabezado por la Presidente de la Nación Cristina Fernández de Kirchner. 
En otra intervención pública ofrecida a un matutino de la Provincia de Buenos Aires Martínez abundó en sus denuncias:

El abierto involucramiento de la inteligencia militar en actividades de espionaje interno, y la presencia cada vez más extendida de efectivos militares a lo largo y ancho del país, en diferentes circunstancias y conflictos, pretende ejercer un efecto disuasivo frente a posibles desbordes sociales provocados por el brutal ajuste por inflación que el kirchnerismo viene ejerciendo salvajemente. (Martínez, 2013, 21 de diciembre)

Máxime a la luz de lo previsto en el artículo 15 de la Ley de Defensa Nacional donde se establece que "Las cuestiones relativas a la política interna del país no podrán constituir en ningún caso hipótesis de trabajo de organismos de inteligencia militar". Con la intención de no excedernos en la presentación de las denuncias efectuadas por figuras políticas, periodistas y especialistas sobre la realización de tareas ilegales por parte de la IM optamos por clausurar la exposición de testimonios, entendiendo que la concordancia entre declaraciones públicas y versiones de fuentes no revelables sientan un suelo de interpretación coherente. Más todavía si el marco estratégico y presupuestario que hace a la composición de lugar dota de sentido contextual a los hechos.

\section{CONCLUSIÓN}

El análisis pormenorizado del conjunto de indicadores tomados en consideración (apreciación estratégica general, rol legal de la IM, modificaciones presupuestarias dentro del GAMIL, información provista por fuentes abiertas y reservadas, distanciamiento entre el PEN y la SI y nombramiento de Milani al frente del Ejército argentino) invita a sustentar la hipótesis que, por el peso de las reflexiones presentada, tomamos como probable a la hora de interpretar la realidad de la IM: el gobierno nacional efectúa inteligencia interna mediante la utilización de algunos segmentos del aparato de IM. Suposición reforzada por la inactividad característica del organismo de control parlamentario de las actividades de inteligencia ${ }^{9}$. Este último dato es

9. Se entiende que la labor parlamentaria de un organismo encargado de ejercer el control de los servicios de Inteligencia (en el caso argentino se trata de la Comisión Bicameral de Fiscalización de las Actividades de los Organismos y actividades de Inteligencia [CB], cuerpo por completo inactivo desde hace años a esta fecha) está mediatizada por cláusulas de confidencialidad como dispositivos tendientes a garantizar los parámetros de secretismo y discreción indispensables en el quehacer de los controles políticos previstos en las leyes vinculadas con el área. Los recaudos detallados se reiteran en todos los casos internacionales conocidos por tratarse de una premisa operativa de carácter programática diseñada en atención a la naturaleza de las actividades llevadas a cabo por parte de la comunidad de inteligencia. Por lo cual, el control parlamentario debe ser riguroso en la ejecución de sus funciones y discreto en lo concerniente a la no divulgación de información sensible para la seguridad nacional. La obtención del difícil equilibrio establecido entre la acción de contralores que velen por la legalidad de la acción de los servicios de Inteligencia 
de especial relevancia como composición de lugar de las acusaciones públicas levantadas por distintas figuras políticas y periodísticas sobre la utilización de la IM como dispositivo ilegítimo de inteligencia interior. Manolo Vela hace hincapié en la problemática de la transparencia como necesidad indeclinable en el propósito de garantizar el funcionamiento de los servicios de inteligencia en un marco socio-político subordinado al imperio de la Constitución. Más todavía Vela vincula los esquemas de contralor deficiente en materia de Inteligencia con designios políticos premeditados por parte del PEN, en dirección a instrumentar el manejo ilegal de los servicios con fines contrarios al estado de derecho:

En el régimen democrático, la labor de control se basa en la acción deliberada que los poderes del Estado realizan a través de una diversidad de instrumentos que se complementan con el ejercicio de derechos ciudadanos en actividades de fiscalización. ¿Cuáles son las razones por las cuales un gobierno electo por el pueblo es incapaz de controlar a sus servicios de inteligencia, aun sabiendo que estos realizan actividades que violan la ley? Teniendo claro que los servicios dependen de los recursos presupuestarios del Estado, la ausencia de estos controles sólo puede ser explicada por la necesidad que el poder político convierta los servicios de inteligencia en piezas de poder (2003).

Huelga decir que en su conjunto las evaluaciones volcadas a lo largo del artículo sólo se prestan a diseñar una apreciación inferencial construida en base a testimonios, opiniones y juicios que distan de organizarse en elementos probatorios positivos. Caso contrario, el canal de comunicación de los hallazgos merecería un encaminamiento judicial destinado a motorizar la destitución de varias personas entre los que actualmente ocupan cargos políticos. Empero, la complejidad y articulación de los hechos atinentes a la situación puesta de relieve conduce por su propia lógica a descifrar los acontecimientos en curso por medio de la hipótesis manifestada. La potencial utilización de elementos pertenecientes a la IM en calidad de instrumento de inteligencia interna vulnera principios democráticos y republicanos nodales en el estado de derecho. El cúmulo de datos traídos a colación invita a, como poco, asignarle verosimilitud a la hipótesis donde derivó el escrito. Va de suyo que la apariencia de verdad no alcanza a transformar la hipótesis en certidumbre. Sin embargo la constatación de un conjunto de elementos de información objetiva en términos estratégicos y presupuestarios complementarios en su sentido con la lectura de los acontecimientos surgida en declaraciones públicas y testimonios

en simultáneo con la circunspección informativa como recaudo estratégico de apariencia anti-democrática, cobra sentido de cara a la identidad de los procedimientos, productos y organizaciones de Inteligencia. En otras palabras: en aras de alcanzar los niveles de eficacia y eficiencia esperados de los organismos de inteligencia en el marco del plexo normativo que rige el sector, el control político debe instrumentarse de forma constante y guardar la discreción requerida. Nuevamente, en el caso de la República Argentina la CB falla por completo en su propósito (Sancho, 2010) ya que no ejecuta actividad alguna a pesar de encontrarse conformada como el único espacio de control político distinto al PEN. Tesitura institucional cuya racionalidad abona desde un costado suplementario nuestra hipótesis sobre la utilización de la IM en actividades ilegales por parte del PEN. 
producidos al amparo de la discreción, le otorga densidad a la aserción esgrimida y sugiere direcciones prospectivas con altos grados de factibilidad. En síntesis, la articulación de los datos relevados en un mismo esquema interpretativo señala la presencia de una desviación ilícita en lo concerniente al desempeño de la IM desde mediados del 2013, y nada en la situación actual insinúa un falseamiento de nuestra hipótesis o reversión alguna en la continuidad de la situación inferida.

\section{REFERENCIAS}

Bartolomé, M. (2006). Seguridad y Defensa en tiempos del bicentenario, Buenos Aires: Instituto de Publicaciones Navales.

(2010). "Si quieres la paz prepárate para... ¿qué?". En M. Bartolomé (Comp.), Seguridad y Defensa en tiempos del Bicentenario (pp. 35-56). Buenos Aires: Instituto de Publicaciones Navales.

Battaglino, J. (2008). Palabras mortales. ¿Rearme y carrera armamentista en América del Sur? Nueva Sociedad, (215), 23-34.

(2013). La Argentina desde 1983: un caso de desmilitarización del sistema político. Revista SAAP 7, (2), 265-273.

Consejo de Defensa Suramericano (2014). Registro suramericano de gastos de defensa. Recuperado de: http://www.ceedcds.org.ar/Espanol/05-DocInf/05-03-Registro-GastoDefensa.html

Crawley, E. (1970). Subversión y seguridad. La cuestión de la guerra de guerrillas en el contexto argentino. Buenos Aires: Círculo Militar.

Decreto N. ${ }^{\circ}$ 727. Reglamentación de la Ley de Defensa Nacional, Boletín Oficial, Buenos Aires, Argentina, 12 de abril de 2006.

Decreto N. ${ }^{\circ}$ 1729. Aprobación para el ciclo de planeamiento para la defensa nacional, Boletín Oficial, Buenos Aires, Argentina, 27 de noviembre de 2007.

Decreto N. ${ }^{\circ}$ 1741. Aprobación de la directiva de política de defensa nacional, Boletín Oficial, Buenos Aires, Argentina, 10 de noviembre de 2009.

Decreto 6703. Aprobación de la estrategia nacional de defensa, Ministerio de Defensa, Brasilia, Brasil, 18 de diciembre de 2008. 
Eissa, S. (2013). Redefiniendo la defensa: posicionamiento estratégico defensivo regional. Revista SAAP, 7(1), 41-64.

Holzmann, G. (noviembre, 2005). Características de un sistema de Inteligencia Estratégica para países emergentes. Análisis y propuesta. Conferencia Sub-Regional de Seguridad Transnacional y Gobernabilidad. Montevideo, Uruguay.

(2010). Post-globlalización y seguridad en una perspectiva regional. En M. Bartolomé (Comp.), Seguridad y Defensa en tiempos del Bicentenario (pp. 201-222). Buenos Aires: Instituto de Publicaciones Navales.

Kent, S. (1968). Inteligencia Estratégica. Buenos Aires: Pleamar.

Ley N. ${ }^{\circ}$ 23.554. Ley de Defensa Nacional, Boletín Oficial, Buenos Aires, Argentina, 26 de abril de 1988.

Ley $N^{\circ}$ 25.520. Ley de Inteligencia Nacional, Boletín Oficial, Buenos Aires, Argentina, 3 de diciembre de 2001.

Morales, G. (2013, 23 de diciembre). "Milani hace tareas de inteligencia interior sobre el periodista Daniel Santoro". Radio continental. Recuperado de http://www.continental. com.ar/noticias/actualidad/gerardo-morales-milani-hace-tareas-de-inteligencia-interiorsobre-el-periodista-daniel-santoro/20131223/nota/2042421.aspx

Martínez, J. (2013, 21 de diciembre). Milani dispondrá de \$1325 millones adicionales para inteligencia y seguridad. Hoy en la noticia. Recuperado de http://diariohoy.net/politica/ milani-dispondra-de-1325-millones-adicionales-para-inteligencia-y-seguridad-19759

(2013, 23 de diciembre). "Milani recibió \$1.325 millones para inteligencia y seguridad interior". Radio continental. Recuperado de http://www.continental.com.ar/ noticias/actualidad/martinez-milani-recibio-1325-millones-para-inteligencia-y-seguridadinterior/20131223/nota/2042397.aspx

Resolución N. ${ }^{\circ}$ 52. Comisión de análisis y evaluación de las responsabilidades políticas y estratégico militares en el conflicto del Atlántico Sur. Informe Rattenbach. Informe revelado por el Decreto 200, Boletín Oficial, Buenos Aires, Argentina, 7 de febrero de 2012.

Sancho, C. (2010). Servicios de Inteligencia en las Américas: estado del debate y desafíos pendientes. En M. Bartolomé (Comp.), Seguridad y defensa en tiempos del Bicentenario (pp. 149-168). Buenos Aires: Instituto de Publicaciones Navales

Senado de la Nación (2013). Proyecto de comunicación, S-4231/13. 
Sepúlveda, I. (2007). La Seguridad Internacional ante las Nuevas Amenazas. En PNUD Argentina - Ministerio de Defensa, Defensa nacional: dimensiones internacionales y regionales. Contribuciones al debate (pp.63-84). Buenos Aires: Programa de Naciones Unidas para el Desarrollo.

Spota, J. (2013). Algunas reflexiones sobre la Defensa Nacional contemporánea. Escenarios Alternativos, 137(1). Recuperado de http://www.escenariosalternativos.org/default.asp?sec cion $=$ escenarios $5 \&$ subseccion $=$ escenarios $5 \&$ nota $=4518$

Szeinfeld, J. (2010). Relaciones cívico-militares en la Argentina. Situación actual. En M. Bartolomé (Comp.), Seguridad y defensa en tiempos del Bicentenario (pp. 321-342) Buenos Aires: Instituto de Publicaciones Navales.

Tello, A. \& Spota, J. (2014). Interrogantes presupuestarios como síntomas de problemas en la Defensa Nacional. Escenarios Alternativos, 141(12). Recuperado de http://www. escenariosalternativos.org/default.asp?seccion $=$ escenarios $5 \&$ subseccion $=$ escenarios $5 \& \mathrm{n}$ ota $=4558$

Ugarte, J. (1999). Legislación de Inteligencia. Especialización y Control, Legitimidad y Eficacia. Buenos Aires: Dunken.

Vanossi, J. (1997). Introducción. El Estado. En Dalla, A.; Graña, E.; Sisinni, N. \& Basterra, M., Manual de Teoría del Estado y del Cobierno (pp. 15-36). Buenos Aires: Editorial de Belgrano.

Vela, M. (2003). La labor de la inteligencia para principiantes. Ciudad de Guatemala: Editorial de Ciencias Sociales.

Vigliero, S. (2010). América Latina frente al Orden Internacional. Desafíos frente al Siglo XXI. En M. Bartolomé (Comp.), Seguridad y defensa en tiempos del Bicentenario. (pp. 81-110). Buenos Aires: Instituto de Publicaciones Navales.

Zúñiga, R. (1970). Introducción. En Crawley, E., Subversión y seguridad. La cuestión de la guerra de guerrillas en el contexto argentino (pp. 7-9). Buenos Aires: Círculo Militar. 